This is a preprint of an article published in Nature Plants. The final authenticated version is available online at: $\mathrm{https}: / /$ doi.org/10.1038/s41477-021-00989-9

\title{
A non-duplicated magnoliid genome
}

Zhen $\mathrm{Li}^{1}$ and Yves Van de Peer ${ }^{1,2,3}$

${ }^{1}$ Department of Plant Biotechnology and Bioinformatics, Ghent University and VIB Center for Plant Systems Biology, Gent, Belgium

${ }^{2}$ College of Horticulture, Academy for Advanced Interdisciplinary Studies, Nanjing Agricultural University, Nanjing 210095, China

${ }^{3}$ Department of Biochemistry, Genetics and Microbiology, University of Pretoria, Pretoria, South Africa.

Almost all flowering plants bear traces of ancient polyploidy. Now, the genome of a magnoliid, Aristolochia fimbriata, is described that is showing no evidence of whole genome duplication (WGD), a feature uniquely shared with Amborella trichopoda, the sister species to all other angiosperms. Since diploidization following polyploidy usually leads to major rearrangements of the genome, the more conserved, ancestral structure of the Aristolochia genome offers great opportunities for comparative genomics.

Ancient polyploidy has been observed in almost all plant lineages and often seems to predate the origin and diversification of major plant lineages ${ }^{1}$. Apart from being considered an important mechanism of speciation and diversification, WGDs increase genetic and biological complexity and retained homeologs (duplicates retained after a WGD) have, for instance, been considered important for the development of flowers ${ }^{2}$ and fruit ${ }^{3}$, and the evolution of biochemical pathways ${ }^{4}$. Consequently, papers describing novel plant genomes with evidence for ancient polyploidy are usually met with some excitement. In a recent paper of Qin and coauthors ${ }^{5}$ on Aristolochia fimbriata, a magnoliid belonging to the order Piperales, excitement on the contrary comes from the lack of a WGD. Of the approximately 200 angiosperm genomes sequenced to date, $A$. fimbriata seems to lack any evidence for ancient polyploidy, beyond the very ancient WGD that predated diversification of all living angiosperms or even seed plants ${ }^{6}$. The only other angiosperm for which this is known to be the case is Amborella trichopoda, a monotypic genus of shrubs or small trees endemic to New Caledonia and believed to be sister to all other living angiosperms (see Figure 1).

Whereas WGDs have great potential for evolutionary innovation, at the same time they greatly complicate the structural and evolutionary analysis of genomes. WGDs are usually followed by massive genome rearrangements during the process of rediploidization, as recently shown again in a comparison between the 'unduplicated' genome of the gnetophyte Gnetum montanum and the genome of Welwitschia mirabilis, which did undergo a WGD7. Genomes such as the one of Gnetum (for gymnosperms) and the ones of Aristolochia and Amborella (for angiosperms) are considered to represent a more ancestral state that can be used to unravel the evolution and genome duplication history of other species. For instance, using the more conserved, ancestral structure of the Aristolochia genome, Qin et al. ${ }^{5}$ show that black pepper (Piper nigrum) has undergone three polyploidy events, and not one, as originally reported ${ }^{8}$. Also, using the Aristolochia genome as a reference, Qin et al. ${ }^{5}$ were able to identify novel WGDs in Piperales and to more accurately determine the timing of previously proposed WGDs in Laurales and Magnoliales.

The 'simpler' Aristolochia genome was also used to revisit the evolutionary relationship of magnoliids. The phylogenetic relationships between magnoliids and the other four mesangiosperm clades, i.e., eudicots, monocots, Ceratophyllales, and Chloranthales, has remained elusive with incongruent branching orders and conflicting topologies of gene trees ${ }^{9-13}$. As only one genome has become available recently for Ceratophyllales and none yet for Chloranthales, phylogenomic analyses have so far mainly focused on the phylogenetic relationships between magnoliids, eudicots, and monocots. Concatenation of multiple, preferentially singlecopy, gene sequence alignments usually either support one of two phylogenies; magnoliids as sister group to a clade of eudicots and monocots ${ }^{10}$, or magnoliids and eudicots forming a clade that is sister to monocots ${ }^{9}$. Although concatenated sequences can eliminate stochastic errors, in some cases even the presence or absence of a single gene can affect the inferred species phylogeny ${ }^{14}$. An alternative phylogenomic approach developed more recently is to infer species phylogenies with gene trees under the multi-species coalescent (MSC) model. 
The MSC model integrates gene genealogies and has been specifically developed to deal with incomplete lineage sorting (ILS). Accumulated evidence has shown that ILS may underlie the discordant gene trees and the incongruent species phylogenies of mesangiosperms because of their rapid radiation, likely accompanied by random sorting of polymorphic alleles into fast-splitting populations ${ }^{11-15}$. Most results based on the MSC model find magnoliids and eudicots as sister to monocots.

In their analysis of the Aristolochia genome, in addition to the phylogenomic analyses described above, Qin et al. ${ }^{5}$ have utilized gene order and content information to infer the phylogenetic position of magnoliids. They first compared the Aristolochia genome with representative genomes of other magnoliids, eudicots, monocots, Nymphaea and Amborella. Using the 'unduplicated', less rearranged, and therefore more conserved genome of Aristolochia, they were able to show that chromosome 7 in Aristolochia corresponds to two separated genomic segments in eudicots, Nymphaea, and Amborella. Interestingly, they could then show that the same pattern holds for all investigated magnoliids and monocots. Since Amborella and Nymphaea are two successively branching sister groups to all other angiosperms (see Figure 1), Qin et al. ${ }^{5}$ proposed that chromosome 7 in Aristolochia represents a derived fusion event shared by magnoliids and monocots. In phylogenetics, such shared derived character is often used to define a monophyletic group, and thus in this case for magnoliids and monocots to share a common ancestor. Interestingly, a similar observation was made recently by Zhao et al. ${ }^{16}$ who also used collinearity and (micro)synteny information to infer phylogenetic relationships within angiosperms. Using a 'character matrix' derived from a network representation of pairwise (micro)synteny relations, and maximum likelihood inference, Zhao et al. ${ }^{16}$ found monocots and magnoliids also to be sister groups, to the exclusion of eudicots, and attributed the clustering of magnoliids and monocots to some syntenic blocks - other than the ones discovered by Qin et al. ${ }^{5}$ - that only seemed to exist in magnoliids and monocots. Again, as in the findings of Qin et al. ${ }^{5}$, alternative explanations could not completely be ruled out, but both findings are worthy of further investigation. In this respect, additional, preferably non-duplicated genomes of Chloranthales and Ceratophyllales would be welcomed.

1 Van de Peer, Y., Mizrachi, E. \& Marchal, K. The evolutionary significance of polyploidy. Nat. Rev. Genet. 18, 411-424 (2017).

2 Zhang, L. et al. The water lily genome and the early evolution of flowering plants. Nature 577, 79-84 (2020).

3 Sato, S. et al. The tomato genome sequence provides insights into fleshy fruit evolution. Nature 485, 635641 (2012).

4 Huang, C. F. et al. Whole genome duplication facilitated the evolution of C4 photosynthesis in Gynandropsis gynandra. Mol. Biol. Evol., doi:10.1093/molbev/msab200 (2021).

5. Qin, L. et al. The Aristolochia fimbriata genome: insights into angiosperm evolution, floral development and chemical biosynthesis. Nat. Plants. (in press).

6 Ruprecht, C. et al. Revisiting ancestral polyploidy in plants. Sci. Adv. 3, e1603195 (2017).

7 Wan, T. et al. The Welwitschia genome reveals a unique biology underpinning extreme longevity in deserts. Nat. Commun. 12, 4247 (2021).

$8 \mathrm{Hu}$, L. et al. The chromosome-scale reference genome of black pepper provides insight into piperine biosynthesis. Nat. Commun. 10, 4702 (2019).

9 Chaw, S. M. et al. Stout camphor tree genome fills gaps in understanding of flowering plant genome evolution. Nat. Plants 5, 63-73 (2019).

10 Chen, J. et al. Liriodendron genome sheds light on angiosperm phylogeny and species-pair differentiation. Nat. Plants 5, 18-25 (2019).

11 Yang, Y. et al. Prickly waterlily and rigid hornwort genomes shed light on early angiosperm evolution. Nat. Plants 6, 215-222 (2020).

12 Chen, Y. C. et al. The Litsea genome and the evolution of the laurel family. Nat. Commun 11, 1675 (2020).

13 Lv, Q. et al. The Chimonanthus salicifolius genome provides insight into magnoliid evolution and flavonoid biosynthesis. Plant J. 103, 1910-1923 (2020).

14 Shen, X. X., Hittinger, C. T. \& Rokas, A. Contentious relationships in phylogenomic studies can be driven by a handful of genes. Nat. Ecol. Evol. 1, 126 (2017).

15 Rendon-Anaya, M. et al. The avocado genome informs deep angiosperm phylogeny, highlights introgressive hybridization, and reveals pathogen-influenced gene space adaptation. Proc. Natl. Acad. Sci. U S A 116, 17081-17089 (2019).

16 Zhao, T. et al. Whole-genome microsynteny-based phylogeny of angiosperms. Nat. Commun 12, 3498, doi:10.1038/s41467-021-23665-0 (2021).

The authors declare no competing interests. 


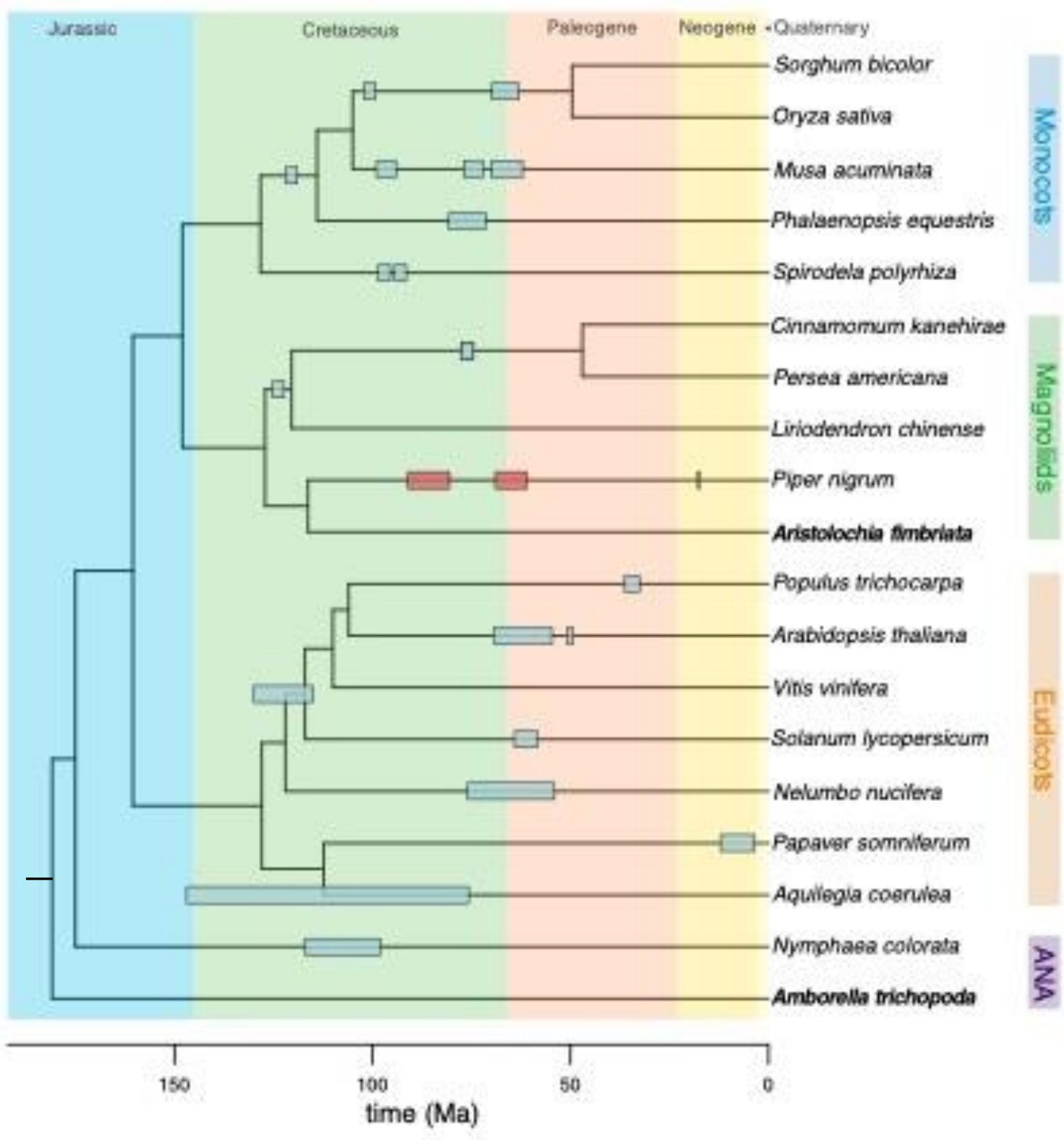

Figure 1. Phylogenetic tree showing some key angiosperms and known whole-genome duplications (WGDs). Phylogenetic relationships as well as divergence times between species are retrieved from TimeTree (http://www.timetree.org). Aristolochia fimbriata ${ }^{5}$ is only the second angiosperm genome - the first one is Amborella trichopoda - known so far that has not experienced a WGD since the divergence of angiosperms. Both species are highlighted in bold. WGDs (with confidence intervals if known) have been placed to the best of our knowledge (and taken from the literature). The two newly discovered WGDs in Piper nigrum ${ }^{5}$ are in red. 